\title{
Early identification of recurrent mood disorders in youth: the importance of a developmental approach
}

\author{
Anne Duffy
}

Department of Psychiatry, University of Calgary, Mathison Centre for Mental Health Research and Education, Canada; acduffy@ucalgary.ca

\begin{abstract}
INTRODUCTION
Adolescence is an important developmental period characterised by accelerated biological, psychological and sociological development. Adolescence is also the high-risk period for onset of major psychiatric disorders that persist into adulthood. In particular, mood disorders in youth account for a substantial proportion of disability and premature death in those aged 10-24 years. ${ }^{1}$ In contrast to prepubertal mood disorders, adolescent mood disorders recur or persist lifelong and are associated with a high estimated heritability. ${ }^{2}$ Suicide is the second leading cause of death in youth in Canada and the UK and is strongly associated with psychiatric disorders; with mood disorders accounting for the greatest proportion. ${ }^{3}$ Therefore, early accurate identification of serious recurrent or persistent mood disorders is of paramount importance, and yet represents a major challenge. This is in part attributable to the overlap of psychiatric symptoms with normative transient distress in this age group, lack of youth friendly specialised mental health programmes and the failure of the current diagnostic approach to consider the natural history of illness development. This paper focuses on the latter problem and specifically highlights evidence from high-risk studies illustrating how a developmental approach, in the context of other risk factors, advances an earlier accurate diagnosis of mood disorders in adolescents and young adults.
\end{abstract}

\section{NATURAL HISTORY OF RECURRENT MOOD DISORDERS IN HIGH-RISK OFFSPRING}

Mood disorders are complex brain diseases that run in families. This is especially true of recurrent melancholic depressive and bipolar disorder, with heritability estimates in the order of $80 \% .{ }^{4}$ The current model is consistent with substantial heterogeneity in multiple predisposing genes that interact with each other and with other risk exposures early in life (including hormones, substance use, early adversity and stress) to determine from among those at familial risk who develops illness and the timing of illness onset. ${ }^{5}$ Epigenetic mechanisms, including DNA methylation and covalent histone modifications within gene promoters, have been shown to be responsive to the environment and in some cases these stable modifications are passed between generations. ${ }^{6}$ Accruing evidence suggests that epigenetic mechanisms may in part explain the 'missing heritability' in these complex disorders. ${ }^{7}$

A confirmed positive family history of recurrent melancholic depression or bipolar disorder in a first-degree family member is the single most robust risk factor predicting the development of mood disorders in young people. ${ }^{8}$ However, the majority of high-risk youth defined by family history alone do not develop mood disorders and the risk actually varies substantially for individuals in different families. ${ }^{9} \mathrm{~A}$ major problem in risk prediction is that there are currently no reliable indicators of illness risk beyond a positive family history. Furthermore, current psychiatric diagnostic approaches do not even consider family history in the formulation, increasing the risk of phenocopies (false positives) and heterogeneity (aetiological). The lack of inclusion of important predictive factors in psychiatry differs from the approach taken in other areas of medicine, where the incorporation of risk factors represents a routine strategy improving early identification and resulting in the development of targeted high-risk early intervention and prevention strategies. Moving forward it will be important to shift from solely syndrome-based diagnoses. The essential platform for studies of illness risk markers must start from a very good understanding of the early natural history of illness development in high-risk youth, ideally based on prospective longitudinal observation.

We are fortunate in this regard in that there are now a number of published and ongoing longitudinal prospective studies of the offspring of parents with confirmed bipolar disorder. ${ }^{10}{ }^{11}$ While there are a number of important differences in included families across the different studies stemming from differences in recruitment, diagnostic methods and loading of psychiatric disorders in adult family members, there are nonetheless complementary consistent findings informing the natural history of recurrent mood disorders in high-risk youth. Specifically, in youth at confirmed familial risk, the predominant presenting mood disorder is a single or recurrent major depressive disorder manifesting in adolescence. $^{12}$ Furthermore, anxiety and circadian disturbances early in childhood appear to be predictive risk syndromes for adolescent-onset mood disorders in high-risk offspring. Specifically, these childhood syndromes have been shown to increase the risk of subsequent mood disorders up to 2.5 -fold in youth at confirmed familial risk. ${ }^{13} 14$

In some clinical and cross-sectional studies of children of affected parents, childhood attention deficit hyperactivity disorder (ADHD) has been proposed as an early risk syndrome. However, longitudinal studies of prospectively assessed offspring of bipolar parents have not generally found ADHD to be a reliable risk syndrome predicting the subsequent onset of bipolar disorder. Rather ADHD in these high-risk children appears associated with other non-specific familial risk factors, and/or limited to a subgroup of high-risk offspring at risk for a psychotic spectrum disorders in others. ${ }^{15}$ Substance use disorders (abuse and dependence) have been shown to manifest early in the course of the evolving mood disorder in high-risk offspring, and represent a substantial early complicating factor associated with worse remission quality, increased hospitalisation and suicide risk and poorer psychosocial and vocational/academic outcomes. ${ }^{16}$ Interesting, emerging evidence suggests that temperament, in the form of high-emotionality and cognitive response style characterised by the propensity to ruminate, may be early psychological indicators of mood disorder risk and as such potentially important early intervention targets in high-risk youth. ${ }^{17} 18$

\section{CLINICAL STAGING AND A DEVELOPMENTAL APPROACH TO DIAGNOSIS}

In an attempt to provide an evidence-based aggregate describing the natural history of recurrent depressive and bipolar disorder in youth at familial risk, Duffy et al ${ }^{13}{ }^{19}$ recently proposed a clinical staging model (figure 1). Clinical staging has been a very useful approach to refining the phenotype along developmental lines in other areas of medicine. Further, understanding the clinical progression of primary recurrent mood disorders holds promise for significant advances in improved early accurate diagnosis, the identification of associated risk factors and biomarkers, and for the development of stage-specific interventions. ${ }^{20}$ Essentially the proposed model includes: Stage 0 (well, but at confirmed familial risk given a first-degree affected relative); Stage 1 (in those at familial risknon-mood childhood disorders including sleep and anxiety disorders, and in some children cognitive deficits/ADHD); Stage 2 (in those at familial risk - minor mood, single episode depression and adjustment disorders 
Only in those at confirmed familial risk

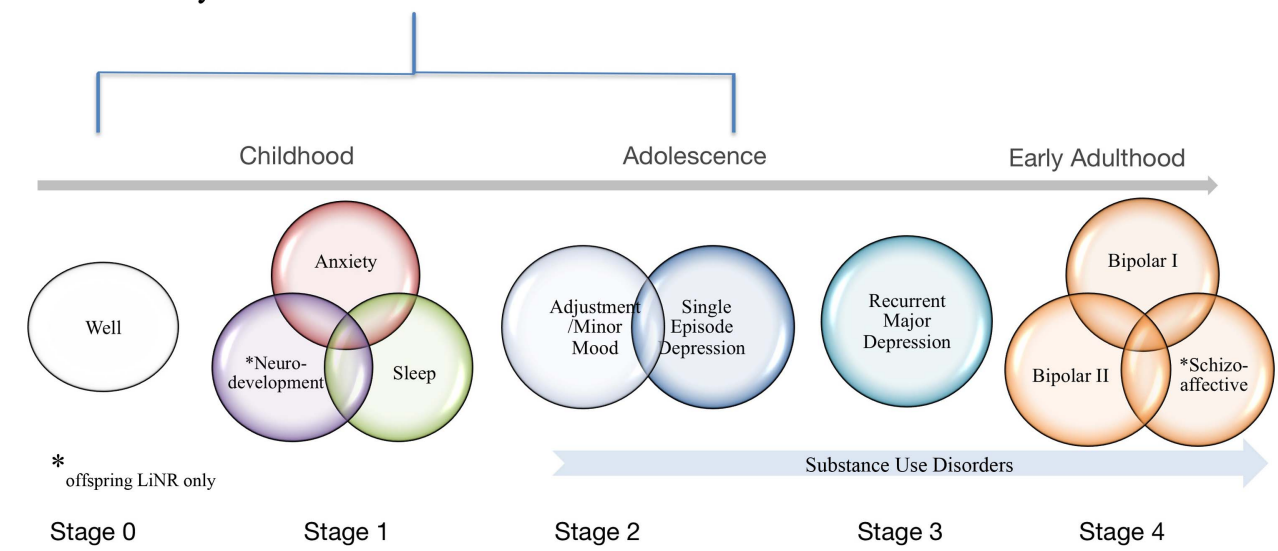

Figure 1 Development clinical staging model of bipolar disorder.

such as anxiety and mood symptoms in association with a stressor around puberty); Stage 3 (recurrent major depressive disorders typically in mid-to-late adolescence); Stage 4 (bipolar or schizoaffective disorder typically late adolescence to early adulthood).

An essential component of this staging model, based on prospective study of high-risk youth, is the limitation of the first few stages (nonspecific but impairing syndromes) to children and youth at confirmed familial risk of developing bipolar disorder or recurrent major depressive disorder. Recurrent major depressive disorder in this context has been shown much more likely related to the bipolar diathesis. Similarly, adolescent onset melancholic depression has been identified as having a higher probability of continuity into adulthood and a higher risk of conversion to bipolar disorder on longitudinal follow-up, especially if characterised by psychotic symptoms, abrupt onset and paradoxical response to antidepressants. ${ }^{21}$ This staging strategy therefore seeks to optimise the early identification hit rate but at the same time limiting over-labelling or the false-positive rate.

Other staging models of bipolar disorder have been proposed but focus on adult patients with already established illness or represent modifications to a staging model that is based on the natural history of psychotic disorders and focuses on the onset of first episode mania (the equivalent to first episode psychosis in these models). ${ }^{22}{ }^{23} \mathrm{~A}$ central problem with these approaches is the neglect of important and predictive early stages or clinical risk syndromes in high-risk youth, occurring years prior to any major mood episodes. By overlooking these early clinical stages in youth at confirmed familial risk, a significant and important opportunity for prevention, early intervention and research aiming to understand the origins of disease are lost. Specifically, anxiety disorders in high-risk offspring have been estimated to onset 8 years on average prior to any diagnosable mood episodes, and depressive episodes have been estimated to onset 4 years on average prior to any diagnosable hypomanic/manic episodes.

The argument for limiting the first few clinical stages to only those youth at confirmed familial risk is to protect against over-labelling and to reduce heterogeneity. For example, prospective studies of youth unselected for family history and manifesting manic-like symptoms have not supported continuity with a bipolar diagnosis in adulthood. Further, epidemiological studies have illustrated that manic-like symptoms are common and typically transient in the adolescent general population. ${ }^{24}$

\section{IMPLICATIONS OF A DEVELOPMENTAL CLINICAL STAGING APPROACH}

Awareness of the limitations of the current syndrome-based diagnostic systems (Diagnostic and Statistical Manual, International Classification of Diseases) and the need to reduce heterogeneity inherent in the resulting broad diagnostic categories is increasing. The need for prospective studies of well-characterised high-risk and patient populations is of paramount importance. These well-characterised high-risk populations are ideally suited to advance our understanding of the nature of the inherited vulnerability, identify genetically sensitive interactive pathways leading to illness onset and to develop effective preventative and early stage acceptable treatments. It is very likely that there are reliable changes at neurological, biological and psychological levels manifesting years prior to any recognisable clinical syndromes in high-risk youth. Unlike psychosis, recurrent mood disorders generally have higher heritability and penetrance across generations. Therefore, a familial high-risk strategy is particularly informative for mood disorder research.

Studies are currently underway to identify vulnerability indicators and markers of illness risk in youth at familial risk of mood disorders. Promising preliminary findings have been reported spanning neuroanatomical markers ${ }^{25}$ to psychological targets ${ }^{26}$ to markers of circadian ${ }^{27}$ and neuroendocrine disturbance to a proinflammatory bias. ${ }^{28}$ The identification of reliable risk indicators will enable the development of an individualised risk assessment model akin to those available for assessing risk of cardiovascular disease and various forms of cancer. This information will also inform the development of novel treatments targeting implicated and interactive biological, psychological and sociological pathways. In the meantime, based on the available evidence and a common sense and 'do no harm' approach, there are certain preliminary recommendations that can be made for youth at high-risk, particularly those that are in the early clinical stages. ${ }^{29}$

The most important requirement for effective prevention and early intervention starts with the development of specialised assessment, treatment and surveillance services targeting high-risk youth. Traditionally, child and adolescent clinical services have been completely separate from adult services. Moreover, child services have largely focused on identifying and treating traditional developmental disorders (autism, learning disabilities, ADHD) and distress of psychosocial origin in young children. However, there remains a major gap in services designed specifically for those at the highest risk of persistent or recurring mood disorders; namely those youth aged 15-25 years. A necessary and welcome change in this direction was the organisation of evidence-based early psychosis programmes spearheaded by the Australian experience. These services are typically organised for youth clinical high-risk of developing psychosis and have been shown to effectively engage this population and reduce the progression to fullblown disorder. ${ }^{30}$ Similar specialised clinical services for youth at risk of or in the early clinical stages of recurrent mood disorders are a needed priority. To this end, integrated collaboration between child and adult services to facilitate the identification and support of children of parents 
with recurrent melancholic depression and bipolar disorder would be an important initiative. This gap represents a major opportunity for change, which would most definitely advance the early intervention clinical and research effort and save lives.

Competing interests None.

doi:10.1136/eb-2014-101993

\section{REFERENCES}

1. Gore FM, Bloem PJ, Patton GC, et al. Global burden of disease in young people aged 10-24 years: a systematic analysis. Lancet 2011;377:2093-102.

2. Rice F. Genetics of childhood and adolescent depression: insights into etiological heterogeneity and challenges for future genomic research. Genome Med 2010;2:68

3. Pritchard C, Hansen L. Child, adolescent and youth suicide and undetermined deaths in England and Wales compared with Australia, Canada, France, Germany, Italy, Japan and the USA for the 1974-1999 period. Int J Adolesc Med Health 2005; 17:239-53.

4. Bienvenu OJ, Davydow DS, Kendler KS. Psychiatric 'diseases' versus behavioral disorders and degree of genetic influence. Psychol Med 2011;41:33-40.

5. Hamshere ML, O'Donovan MC, Jones IR, et al. Polygenic dissection of the bipolar phenotype. Br J Psychiatry 2011;198:284-8.

6. Franklin TB, Russig $\mathrm{H}$, Weiss IC, et al. Epigenetic transmission of the impact of early stress across generations. Biol Psychiatry 2010;68:408-15.

7. Szyf M, McGowan P, Meaney MJ. The social environment and the epigenome. Environ Mol Mutagen 2008;49:46-60.

8. Gottesman II, Laursen TM, Bertelsen A, et al. Severe mental disorders in offspring with 2 psychiatrically ill parents. Arch Gen Psychiatry 2010;67:252-7.

9. Duffy A, Grof P, Robertson $C$, et al. The implications of genetics studies of major mood disorders for clinical practice. J Clin Psychiatry 2000;61:630-7.

10. Duffy A. The early natural history of bipolar disorder: what we have learned from longitudinal high-risk research. Can J Psychiat 2010;55:477-85.

11. DelBello MP, Geller B. Review of studies of child and adolescent offspring of bipolar parents. Bipolar Disord 2001;3:325-34.

12. Duffy A, Alda M, Hajek $\mathrm{T}$, et al. Early course of bipolar disorder in high-risk offspring: prospective study. Br J Psychiatry 2009;195:457-8.

13. Duffy A, Horrocks J, Doucette $\mathrm{S}$, et al. The developmental trajectory of bipolar disorder. Br J Psychiatry 2014;204:122-8.

14. Nurnberger JI Jr, Mclnnis M, Reich W, et al. A high-risk study of bipolar disorder. Childhood clinical phenotypes as precursors of major mood disorders. Arch Gen Psychiatry 2011:68:1012-20.
15. Duffy A. The nature of the association between childhood ADHD and the development of bipolar disorder: a review of prospective high-risk studies. Am J Psychiatry 2012;169:1247-55.

16. Duffy A, Horrocks J, Milin R, et al. Adolescent substance use disorder during the early stages of bipolar disorder: a prospective high-risk study. J Affect Disord 2012;142:57-64

17. Doucette $\mathbf{S}$, Horrocks J, Grof $\mathrm{P}$, et al. Attachment and temperament profiles among the offspring of a parent with bipolar disorder. J Affect Disord 2013;150:522-6.

18. Jones SH, Tai S, Evershed K, et al. Early detection of bipolar disorder: a pilot familial high-risk study of parents with bipolar disorder and their adolescent children. Bipolar Disord 2006;8:362-72.

19. Duffy A, Alda M, Hajek T, et al. Early stages in the development of bipolar disorder. J Affect Disord 2010;121:127-35.

20. McGorry PD. Early clinical phenotypes, clinical staging, and strategic biomarker research: building blocks for personalized psychiatry. Biol Psychiatry 2013;74:394-5.

21. Strober $\mathbf{M}$, Carlson G. Bipolar illness in adolescents with major depression: clinical, genetic, and psychopharmacologic predictors in a three- to four-year prospective follow-up investigation. Arch Gen Psychiatry 1982;39:549-55.

22. Fusar-Poli $\mathbf{P}$, Yung AR, McGorry P, et al. Lessons learned from the psychosis high-risk state: towards a general staging model of prodromal intervention. Psychol Med 2014;44:17-24.

23. Grande I, Magalhaes PV, Chendo I, et al. Staging bipolar disorder: clinical, biochemical, and functional correlates. Acta Psychiatr Scand 2014;129:437-44.

24. Tijssen MJ, van Os J, Wittchen HU, et al. Evidence that bipolar disorder is the poor outcome fraction of a common developmental phenotype: an 8-year cohort study in young people. Psychol Med 2010;40:289-99.

25. Hajek T, Cullis J, Novak T, et al. Brain structural signature of familial predisposition for bipolar disorder: replicable evidence for involvement of the right inferior frontal gyrus. Biol Psychiatry 2013;73:144-52.

26. Pavlickova H, Turnbull 0, Bentall RP. Cognitive vulnerability to bipolar disorder in offspring of parents with bipolar disorder. Br J Clin Psychol 2014;53:386-401.

27. Ritter PS, Marx C, Lewtschenko N, et al. The characteristics of sleep in patients with manifest bipolar disorder, subjects at high risk of developing the disease and healthy controls. J Neural Transm 2012;119:1173-84.

28. Duffy A, Horrocks J, Doucette $S$, et al. Immunological and neurotrophic markers of risk status and illness development in high-risk youth: understanding the neurological underpinnings of bipolar disorder. Int J Bipolar Disord 2014;2:2-9.

29. Duffy A. Interventions for youth at risk of bipolar disorder. Curr Treat Options Psychiatry Published Online First: 14 Jan 2014. doi:10.1007/s40501-013-0006-x

30. McGorry P. Prevention, innovation and implementation science in mental health: the next wave of reform. Br J Psychiatry Supp/ 2013;54:s3-4. 